\title{
¿Por qué publicar un artículo científico?
}

\author{
Why Publish A Scientific Article?
}

La investigación es un proceso importante que mediante la aplicación del método científico permite obtener información relevante que da crédito para entender, verificar, corregir o aplicar el conocimiento. La actividad científica es un modo de ser, pensar, hacer, de construir y de sentir. Un modo de vivir la vida. Tiene que ver con una manera de percibir e interpretar la realidad, una mezcla de razón, conocimiento, pasión, dedicación, emoción, ilusión e intuición, que lo sistematizamos y procesamos en una producción científica difundida para el desarrollo del país como proceso serio y de producción de conocimientos. En esto concurren los esfuerzos personales del investigador (1).

Las investigaciones en salud son una herramienta necesaria para el desarrollo de un país, las cuales deben ser materializadas en políticas públicas que repercutan en el bienestar de la población. En especial los profesionales de enfermería deben prestar atención a innovaciones y actualizaciones que en su campo deben efectuar a fin de crear y mantener una sólida base científica para la práctica profesional que promueva mejores resultados en los cuidados que son la base fundamental del ejercicio profesional de enfermería.

Las universidades son las instituciones con mayor producción de conocimiento científico, por lo que se hace necesario que estos resultados se difundan fuera de ella. La publicación de artículos de investigación muestra la fortaleza que en investigación tiene una Universidad, tal es el caso de la UPCH, la cual es ampliamente reconocida a nivel nacional e internacional por su aporte en investigación, ciencia y tecnología, lo que le permite postular a la obtención de fondos que constituyen el $30 \%$ de su presupuesto. La mejor opción para hacer publicaciones en una revista científica indizada, la Revista de Enfermería Herediana órgano oficial de difusión científica y tecnológica creada y gerenciada por la Facultad de Enfermería de la Universidad Peruana Cayetano Heredia, cumple ese requisito (2).

Los artículos que se presentan en ella pueden ser trabajos originales, artículos de revisión, comunicaciones cortas, estudios de casos entre otros.

La Facultad de Enfermería de la Universidad Peruana Cayetano Heredia, se dedica a impulsar la actividad investigativa en las áreas de pre y postgrado, debido a que la investigación es una responsabilidad científica indispensable para la enfermera en cualquiera de sus campos de actuación logrando así alimentar el proceso de aprendizaje.

Por lo anteriormente expuesto, nos hacemos la pregunta ¿Por qué publicar un artículo científico?

La publicación de un artículo científico contribuye al desarrollo de la sociedad y a que otros investigadores acorten sus tiempos de investigación. El científico no se hace célebre por hacer un descubrimiento, lo hace cuando divulga el mismo en una reconocida fuente de información y en el menor tiempo, lo cual es posible publicando los resultados de su investigación en una forma adecuada, en una revista científica (2). 
Por otro lado; permite que los resultados sean utilizados por otros investigadores; muchos investigadores se conforman con obtener resultados y guardarlos privando así el acceso a otras personas interesados en el tema y haciendo inútil el esfuerzo, tiempo y dinero empleado en realizar el estudio de campo. La tarea del investigador no termina cuando finaliza la fase de campo de su experimento. El trabajo estará concluido cuando otros investigadores puedan hacer uso de los resultados y de los métodos empleados en la investigación.

El investigador que publica siente satisfacción de ver publicado el fruto de su esfuerzo, eleva su prestigio profesional, y se da a conocer ante la comunidad científica. Por medio de publicaciones los autores pueden recibir reconocimientos que aumentan su renombre.

Se puede afirmar que publicar en la revista de Enfermeria de la Universidad Peruana Cayetano Heredia beneficia a todos. Adicionalmente, teniendo en cuenta que la revista es un espacio de divulgación para los grupos de investigación tanto de la Facultad como de otras universidades de carácter nacional e internacional, los artículos publicados enriquecen las discusiones académicas en las comunidades académicas e investigativas tanto de nuestra institución como de otras instituciones.

La revista de Enfermeria Herediana da la bienvenida a los trabajos de investigación que nos hagan llegar enfermeros y estudiantes de nuestra universidad y de otras instituciones nacionales o extranjeras porque ellos son la razón de nuestro trabajo.

Mg. Velásquez Carranza Doris

\section{REFERENCIAS BIBLIOGRÁFICAS}

1. Rojas L; ¿Por qué publicar artículos científicos?; Revista Científica Electrónica de Ciencias Humanas; Venezuela; 2008; Disponible en: http://www.revistaorbis.org.ve/pdf/10/Doc1.pdf

2. Ardito F; El Artículo Científico y la importancia de su publicación; Universidad Peruana Cayetano Heredia; Perú; 2012; Disponible en: http://www.unsaac.edu.pe/vrin/archivos/13articulo.pdf

$1 \quad$ Facultad de Enfermería, Universidad Peruana Cayetano Heredia. Lima, Perú. 